\title{
Histopathological changes in the reproductive system (ovaries and testes) of Oreochromis mossambicus following exposure to DDT
}

\author{
Sibonani S. Mlambo ${ }^{\mathrm{a}, *}$, Johan H.J. van Vuren ${ }^{\mathrm{a}}$, Irene E.J. Barnhoorn ${ }^{\mathrm{b}}$, Maria S. Bornman ${ }^{\mathrm{b}}$ \\ ${ }^{a}$ Department of Zoology, University of Johannesburg, Auckland Park 2006, \\ South Africa ${ }^{b}$ School of Medicine, Department of Urology: Andrology, \\ University of Pretoria, South Africa
}

\begin{abstract}
A b s t r a c t
This study assesses the effects of 1,1-bis (4-chlorophenyl)-2,2,2-trichloroethane (DDT) on the reproduction and gonadal histology of adult Mozambique tipalia (Oreochromis mossambicus). The fish were allowed to breed, following exposure to 2 and 5 jig/l of waterborne technical-grade DDT for 40 days. Fertilized eggs were artificially incubated. In the 5 jig/l exposure, posthatch survival was significantly lower, and prevalence of larval skeletal deformities significantly higher, compared to the control $(p<0.05)$. Incomplete axial development was the common gross deformity in posthatch larvae, caused by failure to develop chondroblasts posterior to the buccopharyngeal cavity. There were no significant differences in the gonadosomatic index of exposed and non-exposed male and female adults. The exposure caused increased oocyte atresia in the ovaries and disorganization of seminiferous lobules in the testes of adults. DDT exposure reduced survival and increased deformities in larvae, at levels that did not cause severe histopathological changes to parental gonads.
\end{abstract}

\section{Introduction}

1,1-Bis (4-chlorophenyl)-2,2,2-trichloroethane (DDT) is a persistent pesticide, whose compound structure permits several different isomeric forms, and has been implicated as an endocrine disrupting chemical (EDC) (Arcand-Hoy and Benson, 1998). Technical-grade DDT contains $65-80 \%$ p,p'-DDT, $15-21 \%$ o,p'-DDT and up to $4 \%$ p,p'-DDD (bis[4-chlorophenyl]-1,1,-dichloroethane) (ATSDR, 2002). The o,p-isomer constitutes about 15\% of technical-grade DDT, and has been reported to be weakly estrogenic (Donohoe and Curtis, 1996; Metcalfe et al., 2000; Papoulias et al., 2003). DDTisomers, including o,p'-DDT have been implicated in reduced development of penises in male alligators from a contaminated Lake Apopka, Florida, USA (Guillete et al, 1994). In a study where o,p'-DDT was administered intraperitonially in male Nile tilapia, it was shown that it has the potential to disrupt the endocrine system of whole fish (Lean os-Castañeda et al., 2007). The p,p'-isomers of DDT are considered androgen receptor antagonists (Kelce et al., 1995).

In 2000, South Africa negotiated to continue with limited and controlled application of DDT to eradicate the pyrethroid resistant mosquito Anopheles funestus and thus reduce the incidence of malaria in affected regions (Bouwman, 2002). There have been several reviews on the toxic effects and health risks of DDT in humans and in wildlife (Turusov et al, 2002; Dalvie et al., 2004; Rogan and
Chen, 2005). Observed effects of DDT on wildlife have ranged from subtle changes in basic physiology of species to permanently altered sexual differentiation (Edmunds et al., 2000). Recent field surveys carried out in the Rietvlei Nature Reserve in South Africa showed the presence of DDT residues in the water and sediment (Bornman et al., 2007).

Histopathology has received increased interest as an endpoint in EDC research in aquatic organisms, because histopathological changes are often the result of the integration of a large number of interactive physiological processes. Sub-lethal exposure to persistent organic compounds may alter key enzymatic activities and potentially cause reproductive effects, by reducing fecundity and population recruitment (Patyna et al., 1999). The hypothalamic-pituitary-gonadal (HPG) axis of teleost fishes, whose principal components are the hypothalamus and pituitary gland in the brain, the gonads and the liver, is generally similar to that of other oviparous vertebrates (Ankely and Johnson, 2004). The gonads are the primary organs of reproduction and, as such, will reflect substantive disturbances to the HPG axis (USEPA, 2006). Histopathology helps to identify target organs of toxicity and mechanism of action (Wester et al., 2002). As a tool for assessing endocrine disrupting effects in fish, histopathology has also been applied in other studies such as endocrine disruption in the ovaries and testes of zebrafish (Van der Ven et al., 2003) and in the adult fathead minnow (Leino et al., 2005).

Toxicologic histopathology is used to identify and describe morphologic differences between unexposed and compound-exposed animals. The Mozambique tilapia (Oreochromis mossambicus), was 
used in this study as a bioindicator of the histological effects of DDT on the gonads, and to observe indications of transmission of effects to the offspring. It was selected for this study because it can spawn repeatedly several times during the year. There is also the deposition of clutches of numerous eggs which can be fertilized by the same male, and this assures ample numbers of genetically similar offspring that are incubated under identical conditions (Holden and Bruton, 1992).

Whole organism endpoints included in the assessment of reproductive effects in this study were fecundity (number of eggs spawned), hatching success (number of eggs that produce larvae) and larval viability which in this case was the survival of the larvae beyond full yolk absorption and the occurrence of malformations in the larvae. This type of information has ecological relevance in that it can be useful for subsequent population extrapolation in ecological risk assessment (Wester et al., 2002; Hutchinson et al., 2006). The aim of this study was to assess gonadal histopathological changes associated with aqueous DDT exposure and effects on reproduction success. This study further contributes to the development of $O$. mossambicus as a model species for EDC testing as it has already found many applications in EDC testing (Marchand et al., 2008; Hill, 2009).

\section{Materials and methods}

\subsection{Test organism}

The fish were obtained from the University of Zululand aquarium in Richards Bay, South Africa, where they had been bred under laboratory conditions. The fish were acclimated for 4 weeks in $1000 \mathrm{l}$ tanks receiving continuous flow of dechlori-nated water at $25-28{ }^{\circ} \mathrm{C}$ (Van Dyk et al., 2007). The sub-lethal DDT exposures were conducted at nominal concentrations of 2 and 5/jig/l of 1,1-bis (4-chlorophenyl)-2,2,2-trichloroethane (DDT) (Sigma). These nominal concentrations were selected in the context of field measurements from a survey done in an urban reserve in South Africa (Bornman et al., 2007). The concentrations also fall within the sub-lethal range that has been tested by other authors (Benton et al., 1994; Metcalfe et al., 2000; Cheek et al., 2001). The fish were kept under a 12:12 light:dark (LD) cycle regulated by electric timers throughout the exposure period, and the water temperature at $25-28{ }^{\circ} \mathrm{C}$. The control and exposure fish were fed every other day to satiety between 9 and $10 \mathrm{a} . \mathrm{m}$. on trout pellets. The organisms were fed at regular and consistent intervals, as it has been noted that inadequate nutrition can adversely affect the GSI, egg production and basal metabolism as much as xenobiotics (Patyna et al., 1999).

A solvent control containing ethanol and a blank with no DDT were run concurrently with the exposures. Water quality parameters were checked weekly and the following values were recorded and are presented here as meaniS.D.: temperature $26.9^{\circ} \mathrm{C} \pm 1.1 .3$; $\mathrm{pH} 6.97 \pm 0.16$; conductivity 192.94 (xS/cmi43.05; oxygen saturation $88.5 \% \pm 5.58$; total dissolved solids $105.88 \mathrm{ppm} \pm 5.76$.

\subsection{Exposure system}

The test solutions were prepared first from a concentrated solution of 1,1-bis (4-chlorophenyl)-2,2,2-trichloroethane dissolved in $10 \mathrm{ml}$ of absolute ethanol. A 500 JULl aliquot of each stock solution was required to be added to $900 \mathrm{ml}$ of gently aerated reverse osmosis water. The exposures were carried out in an adaptation of the semi-static renewal system renewed every 7 days. The average measured concentrations at the end of each 7-day exposure cycle were measured and found to be below detection limit for the blank, the solvent control and 2(jLg/l; and for 5/jig/l were $0.15(\mathrm{jg} / 1$ 4,4-DDD; 0.1 (xg/1 2,4-DDT. The water analysis was done at The Residue Laboratory of Agriculture Research Commission at Onderstepoort Veterinary Institute (South Africa) using GC coupled to a quadrupole MS detector, Standard Method GCMS 003.

\subsection{Exposure procedure}

The fish were of a mean length of $19.4 \mathrm{~cm}$ (S.D. \pm 2.34 ) and a mean mass of $99.8 \mathrm{~g}$ (S.D. \pm 33.7 ). The exposures commenced with sexually mature adult fish $n=3$ males and $n=6$ females, to allow the organisms breeding space because sexually mature male $O$. mossambicus exhibit aggressive territorial behaviour. The fish were kept underexposure conditions foratotalof40days in separate sections of the tank. The chronic exposures were intermittent to avoid overwhelming the fish. After every 7 days of exposure the fish were kept in re-circulating water with no DDT for $24 \mathrm{~h}$. After 40 days the exposure was discontinued and the fish placed in a re-circulating untreated water system. The fish were allowed to breed in reverse osmosis (RO) water that did not contain any chemicals. Hence the fertilization and transfer of eggs into the female's mouth occurred in untreated water and the eggs did not have direct contact with water treated with DDT. Solvent control treatments were carried out under the same conditions, using $500 \mathrm{JULl}$ of absolute ethanol in $900 \mathrm{ml}$ of RO water. Water discharged from the exposure tanks was filtered through activated carbon before disposal.

\subsection{Breeding and fertilization success}

After spawning the organisms were carefully removed from the tank using a hand-net. The fertilized eggs were manually extracted from the female's mouth by gently opening its mouth into a bowl of RO water. The eggs were counted and placed in an incubator that was designed to enable water circulation and aeration. This simulated the movement of gill-plates in the female which provide gentle tumbling and water circulation for the eggs in the mouth. There was no further exposure of the eggs and they were incubated in uncontaminated RO water. The number of successfully hatched eggs was noted. At 10 days posthatch (dph) the larvae were sampled for histological analysis to analyse differences in the interior structure of the larvae with deformities and those that were not deformed.

\subsection{Sample preparation}

The sex, gutted body weight and length of the breeding fish were recorded. The fish were killed by severing the spine just below the head(Du Preezetal., 1997). The gonads were re moved and weighed to the nearest mg then expressed as a percentage of total gutted body weight to obtain the gonadosomatic index (GSI).

\subsection{Histological preparation}

The gonads of adults were fixed in Bouin's Fixative for $18 \mathrm{~h}$ and stored in $70 \%$ methanol.The 10 dph larvae were killed instantly by placing in ajar with a few drops offormalinthenthey were fixed in $10 \%$ neutrally buffered formalin. Dehydration was carried out in an ascending series of ethanol and the tissue was cleared in xylene. The tissues were then embedded in paraffin wax and cut into $5 \mid \mathrm{xm}$ sections on a rotary microtome. The sections were mounted on glass slides and stained with haematoxylinH\&E(VanDyket al., 2007). Histological sections of 10 dph larvae were taken from the anterior abdominal cavity (Gisbert et al., 1998).

\subsection{Histopathology}

The general morphological and histological structures of $O$. mossambicus gonads have been studied in detail, and six stages of sexual maturity have been described by Hatikakoty and Biswas (2004). The organisms in this study were dissected in pre-spawning to spawning phase. Semi-quantitative analysis of gonadal histological changes in the exposed breeding organisms was done on an arbitrary scale of $1-5$, relative to how widespread the effect was within a single specimen. Therefore 0 represented a complete absence of lesions and 5 the most widespread lesion or structural alterations. Due to the subjective nature of interpretations forhistopatho-logic effects caused by toxicants, observations were limited to specific and clearly distinguishable disturbances that can be verified by different investigators (USEPA, 2006). The ovaries were examined for atresia and other changes related to atresia. The testes were analysed for changes in the organization of seminiferous lobules and the appearance of the cells associated with different stages of spermatogenesis.

\subsection{Statistical analysis}

The results are presented as mean \pm S.E. Statistical analysis was by one-way analysis of variance (ANOVA) and Dunnett's Multiple Comparison Test was used to determine significant difference against the control.

\section{Results}

\subsection{Egg counts and hatching success}

Egg counts were expressed in proportion to body weight (relative fecundity) (Fig. 2) because independently the total egg counts did not show a pattern, and seemed to suggest that absolute fecundity was lowest in the blank (Fig. 1). There was a lower number of eggs hatched in the $5 \mathrm{u.g} / \mathrm{l}$ group compared to the control but the difference was not statistically significant (Fig. 1). There was no significant difference in the number of eggs/100 g of body weight between the exposed groups and the control (Fig. 2).

Posthatch survival was given as a percentage the total number of eggs spawned at the beginning. The eggs that were hatched with malformations did not survive beyond full yolk absorption. Fig. 3 shows that survival in the 5 u.g/l exposure was the only one significantly lower than the control. 


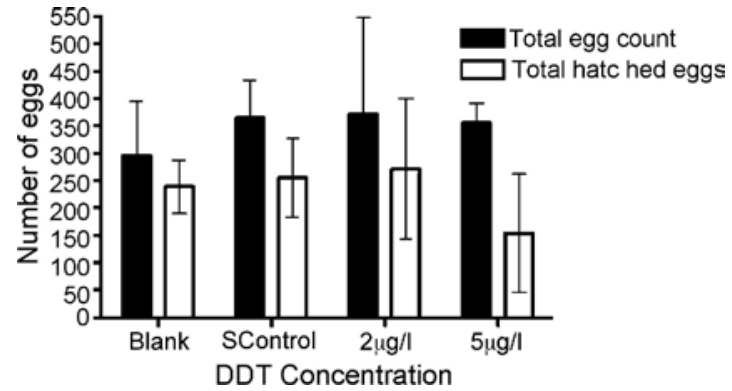

Fig. 1. Mean total egg count (absolute fecundity) and mean total number of eggs hatched. Error bars represent \pm S.D. The total number of eggs hatched was lowest in the $5 \mathrm{jLg} / \mathrm{l}$ but not statistically significant. Scontrol $=0.5 \% \mathrm{v} / \mathrm{v}$ ethanol.

\subsection{Deformities}

The gross deformity that was observed in these experiments was incomplete axial development in the offspring. Histological assessment showed that in the deformed larvae there was insufficient development of chondroblasts and myoblasts posterior to the buccopharyngeal cavity below the brain (Schilling and Kimmel, 1997). Fig. 4 shows that the most significant number of deformities occurred in the $5 \mathrm{jig} / \mathrm{l}$ exposures. The deformities were observed in the control group, but were significantly higher in the $5 \mathrm{jig} / \mathrm{l}$ group.

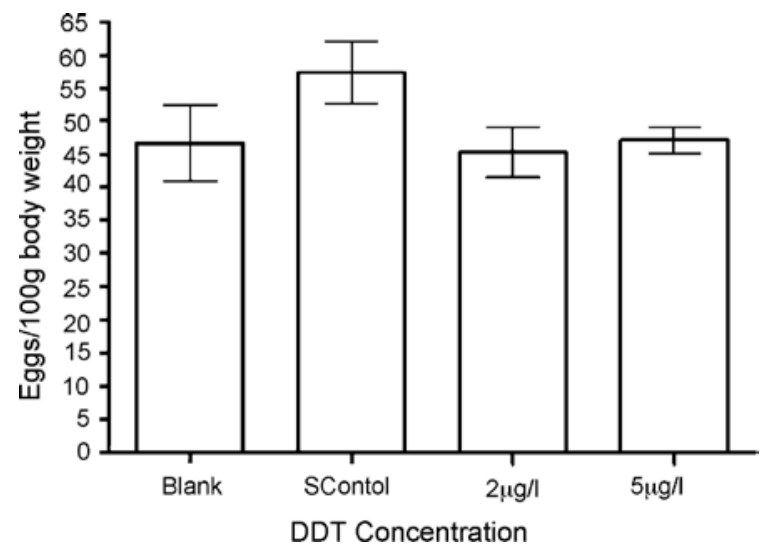

Fig. 2. Relative fecundity of exposed and non-exposed female O. mossambicus. Presented as mean eggs per $100 \mathrm{~g}$ of body weight, error bars represent \pm S.D. Not statistically significant compared to the control $p>0.05$, d.f. $=3$. Scontrol $=0.5 \% \mathrm{v} / \mathrm{v}$ ethanol.

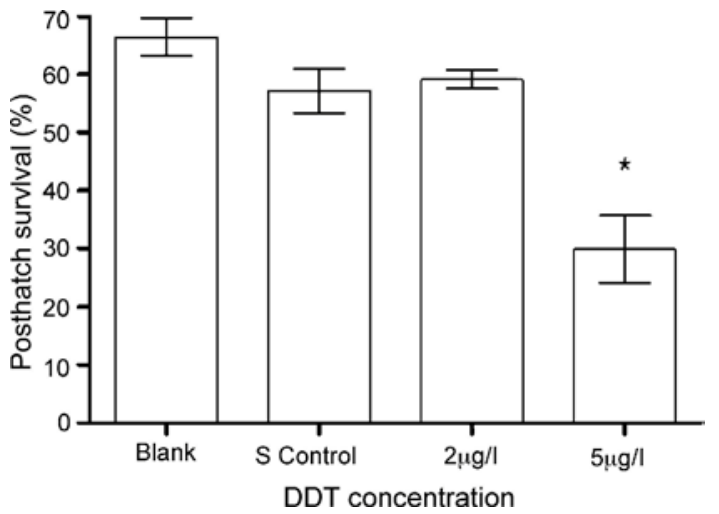

Fig. 3. The number of larvae able to survive past the hatching stage under artificial incubation, following exposure of parents. Analysis done on arcsin-transformed percentages of the total number of eggs spawned. The $5 \mathrm{jLg} / \mathrm{l}$ was significantly higher than all the other three treatments at $p<0.05$ by one-way ANOVA. The graph represents the mean \pm S.E., d.f. $=3$. *Significant difference from the control group by Dunnett's post hoc comparison.

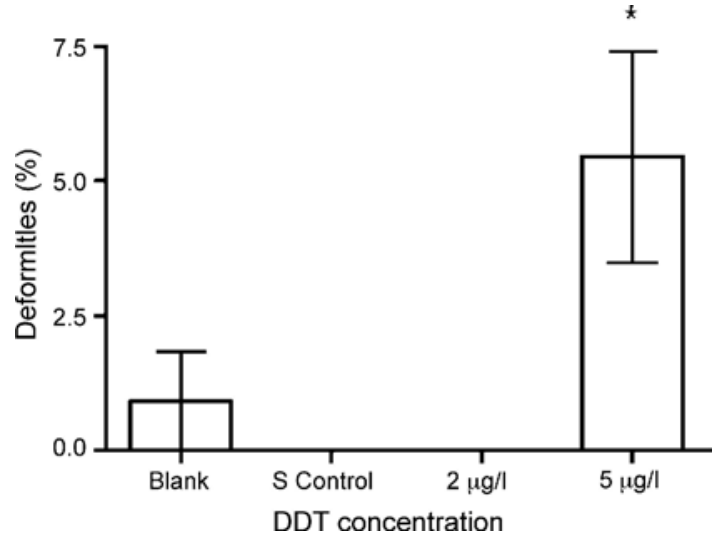

Fig. 4. The mean percentage of deformities in the F1 posthatch larvae after water-borne DDT exposure of parents. Deformities are arcsin-transformed value of percent deformities. Scontrol $=$ solvent control $0.5 \% \mathrm{v} / \mathrm{v}$ ethanol. *Significant difference from the control group using Dunnett's post hoc comparison $(p<0.05)$, d.f. $=3$.

\subsection{Gonadosomatic index}

The GSI was calculated from adult males and females following spawning after a 40-day exposure period. Fig. 5 shows that there were no significant differences in GSI between the organisms exposed to DDT and the controls.

\subsection{Histopathology of the ovaries}

A score of zero was assigned if no atretic oocytes were observed. Atretic oocytes were characterised by loss of oocyte shape, a distorted chorion and a disorganized and fragmented yolk (Hall et al., 2007) (Fig. 9). The breakdown of granulosa layers (which maintain the structural integrity of the oocyte) leads to structural impairment and functional loss. The occurrence of atresia was higher in

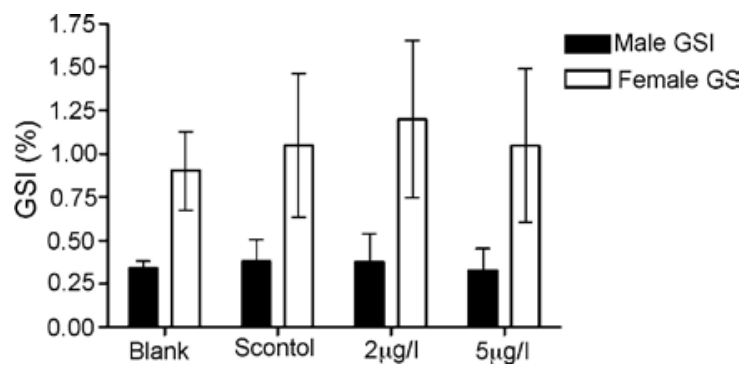

Fig. 5. The GSI of adult male and female O. mossambicus following exposure to DDT There was no statistically significant difference between the GSI of exposed and control organisms. Graph represents the mean \pm S.E., d.f. $=3$.

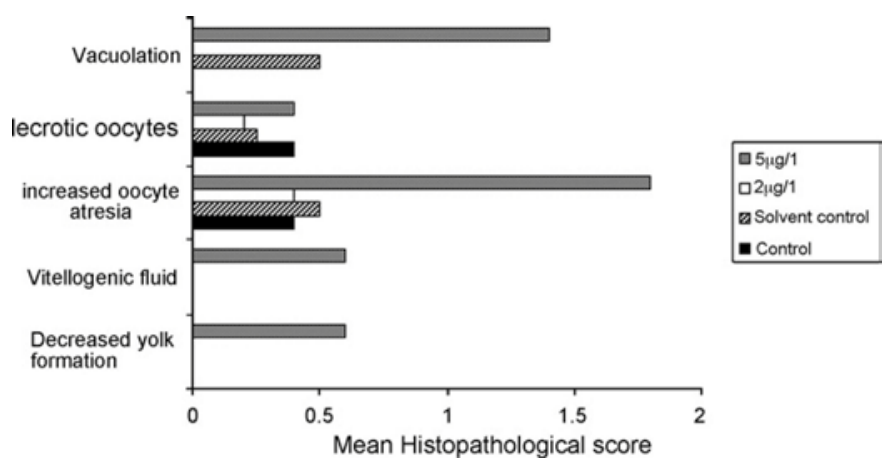

Fig. 6. The mean histological score of the ovaries of adult $O$. mossambicus exposed to DDT. The highest frequencies are shown for $5 \mathrm{jLg} / \mathrm{l}$ but on a 1-5 grading scale the mean frequencies were all below 2 . 


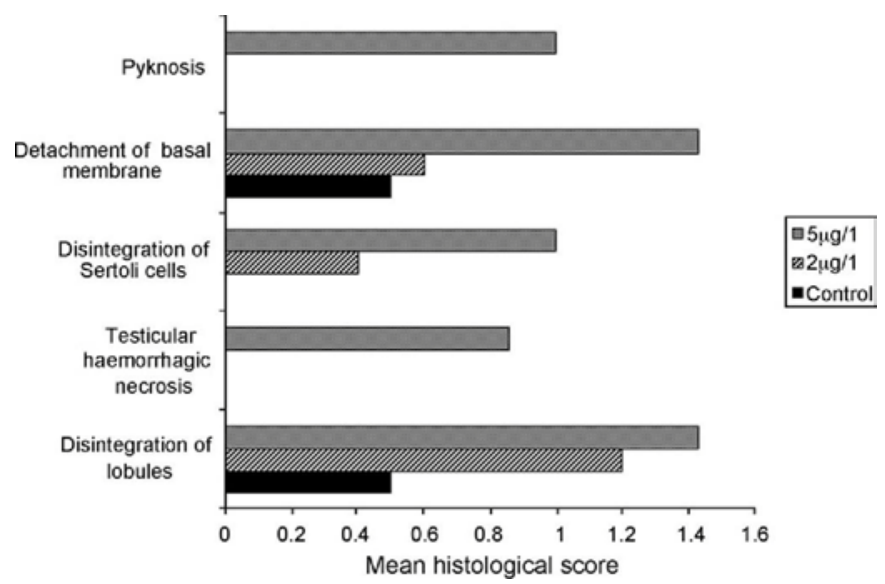

Fig. 7. The mean histological score of the testes of adult $\mathbf{0}$. mossambicus exposed to DDT. The highest frequencies are shown for 5 (xg/l but on a 1-5 scale the mean frequencies were all below 1.5. The grading was according to how widespread an effect is in a single specimen.

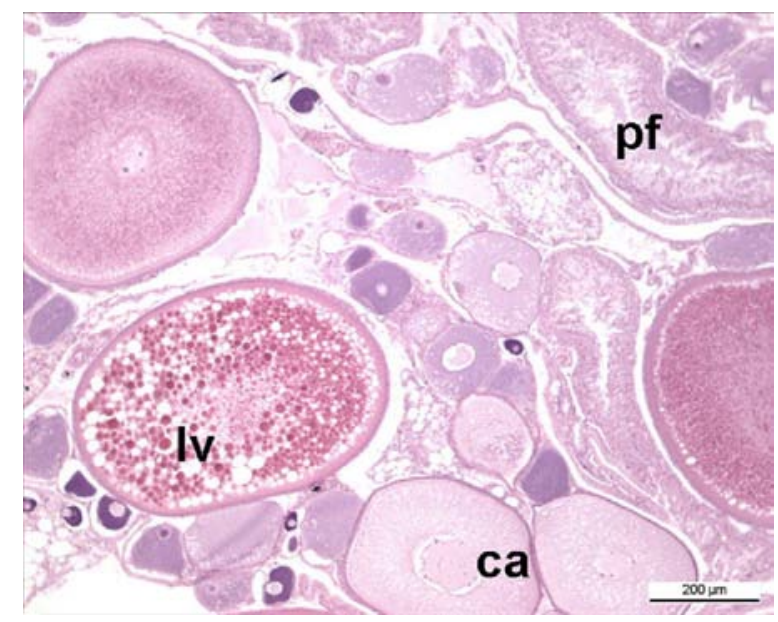

Fig. 8. Ovary from a an adult female $O$. mossambicus from a control group showing ca-cortical alveolus stage oocyte; lv-late vitellogenic stage oocyte with a large numberofyolk globules thatfill the entire ooplasm; pf-post-ovulatory follicle. H\&E stain.

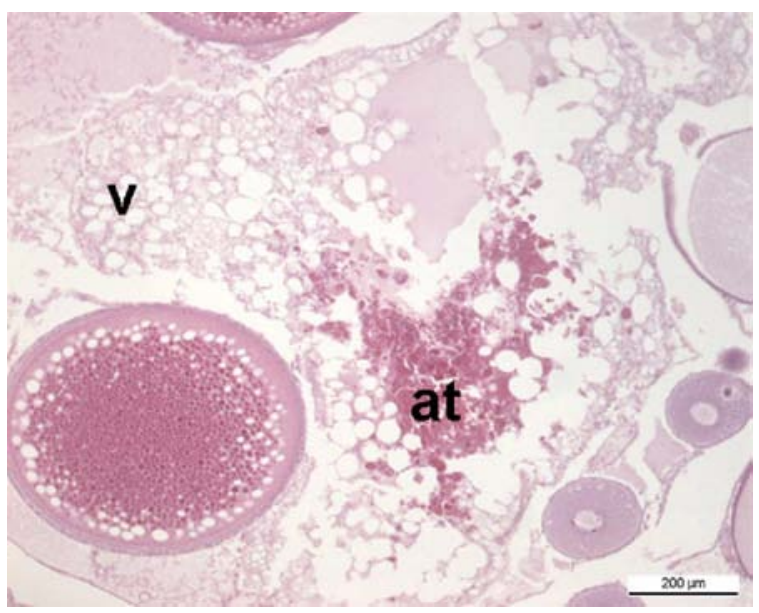

Fig. 9. Ovary from a spawning female 0 . mossambicus exposed to 5 (xg/l showing oocyte atresia (at) and vacuolation (v). H\&E stain.

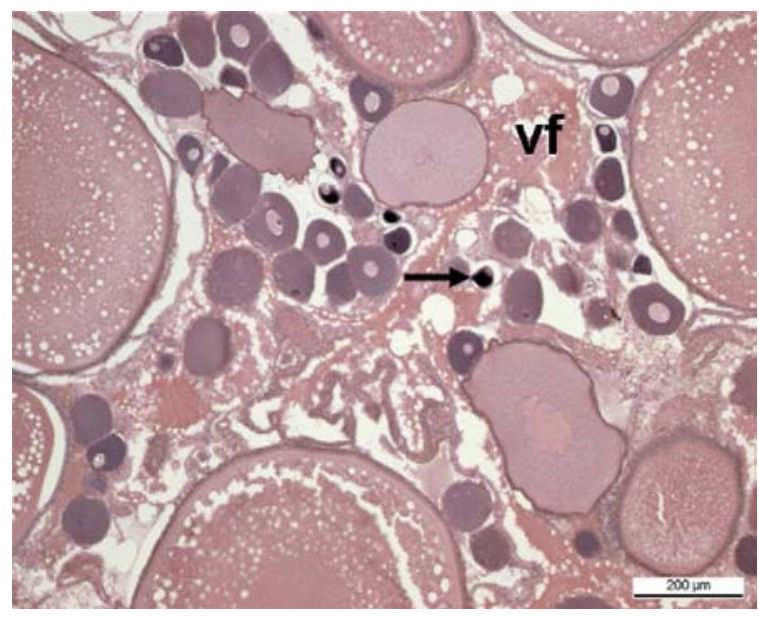

Fig. 10. Ovary from an adult female 0 . mossambicus from the 5 (xg/l exposure stained with H\&E Presence of vitellogenic fluid (vf) inthe ovarian parenchyma, possibly from degeneration of mature vitellogenic oocytes. Necrotic oocyte (arrow).

the 5 ljig/l exposure than the control (Fig. 6). The appearance of undisturbed vitellogenic oocytes and cortical alveoli is shown in Fig. 8, where there is also no vitellogenic fluid in the interstitial space.

A score of 0 was assigned if no distinct vacuoles were observed within the mature oocytes or interstitial tissue. Vacuolation was not recorded in the 2 u.g/l and control samples. Vitellogenic fluid was observed as a pink dark fluid filling the interstitial space or the ovarian parenchyma (Fig. 10) and this was possibly a result of degeneration of mature vitellogenic oocytes. A score of 0 was assigned for samples with no indication of vitellogenic fluid. Fig. 6 shows that this only occurred in some $5 \mathrm{u.g} / \mathrm{l}$ samples and not in other exposure groups.

When a cell has sustained irreversible damage a succession of morphological changes occur which are grouped under the term cell necrosis. Necrosis is pathological cell death, whereby irreversible change occurs due to irreparable cell injury. Cell death which was perceived to be necrosis was observed in the early stage oocytes, mainly the early and late primary oocytes (Fig. 10). Necrotic oocytes were not prevalent, and a score of 0 represented complete absence of necrotic oocytes in the ovary. Necrosis of primary oocytes was observed in the control group as well as in the organisms exposed to DDT (Fig. 6).

\subsection{Histopathology of the testes}

Spermatogonia undergo numerous mitotic divisions to form primary spermatocytes, secondary spermatocytes, spermatids and spermatozoa; resulting in production of cysts surrounded by Sertoli cells. Each lobule of the testes contains numerous cysts. Regularly organized cysts with all stages of spermatogenesis are an indication of testes in a healthy reproductive condition (Pikulkaew and Wongsathein, 2006). Out of a possible maximum severity score of 5, the mean histological scores in the testes were all below 1.5 (Fig. 7). Fig. 7 also shows that histopathological effects were observed mainly in the exposed organisms and not in the control. Fig. 11 shows epithelial lifting of the basal membrane in a spawning male where blood capillaries become conspicuous and seminiferous lobules become larger and full of sperm. Figs. 12 and 13 highlight disorganized lobules where the cysts structures appear to be disrupted as shown by the arrangement of the cells, and there is loss of supporting Sertoli cells. Fig. 13 shows pyknosis condensation of the nuclei in the spermatocytes. This was observed in only one specimen, from the $5 \mathrm{u.g} / \mathrm{l}$ exposure. Testicular haemorrhagic necro- 


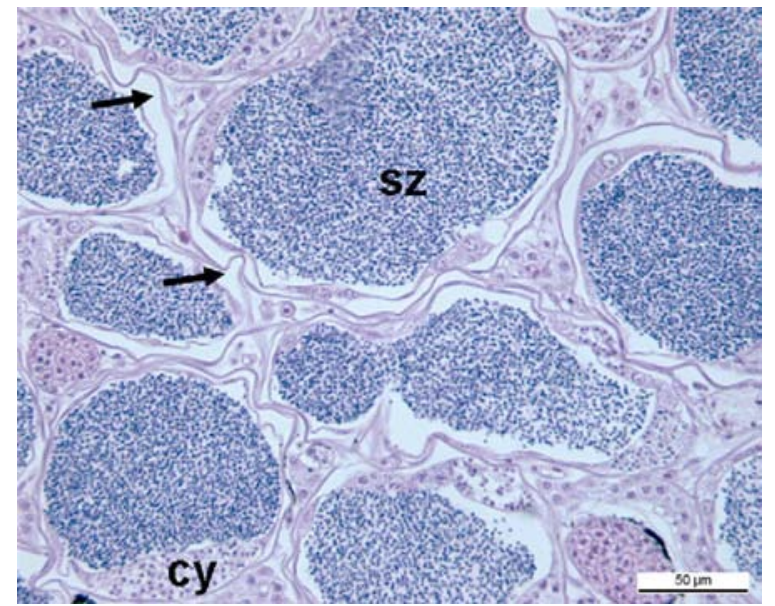

Fig. 11. Sectionofthe testesofanadult maleO. mossambicus from the control group. The figure highlights areas with epithelial lifting of the basal membrane (arrow); the appearance of an undisturbed cyst (cy) containing germ cells at same stage of development; spermatozoa (sz) which are released into the lobular lumen before being channelled to the sperm duct.

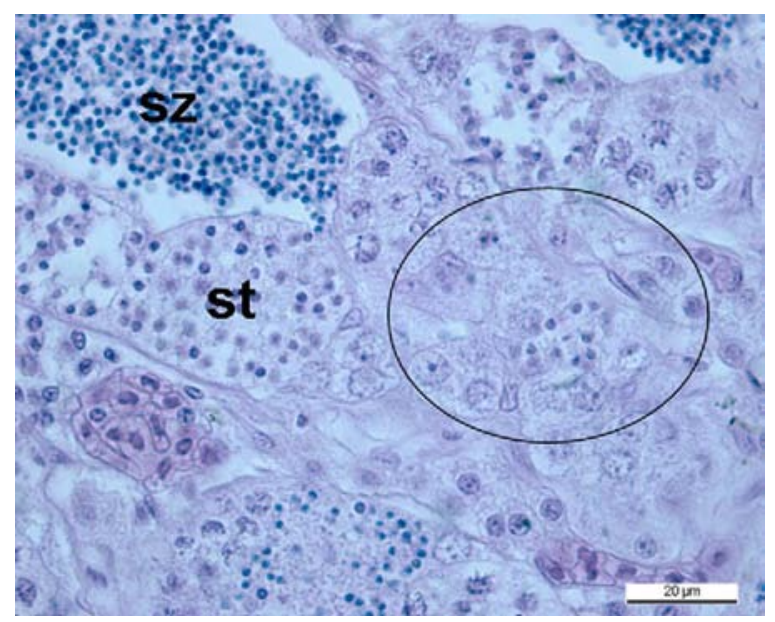

Fig. 12. Longitudinal section of the testes of a male $\mathbf{0}$. mossambicus from the 5 (xg/l exposure; stained with H\&E; st-spermatids. The circle shows a disorganization of cysts.

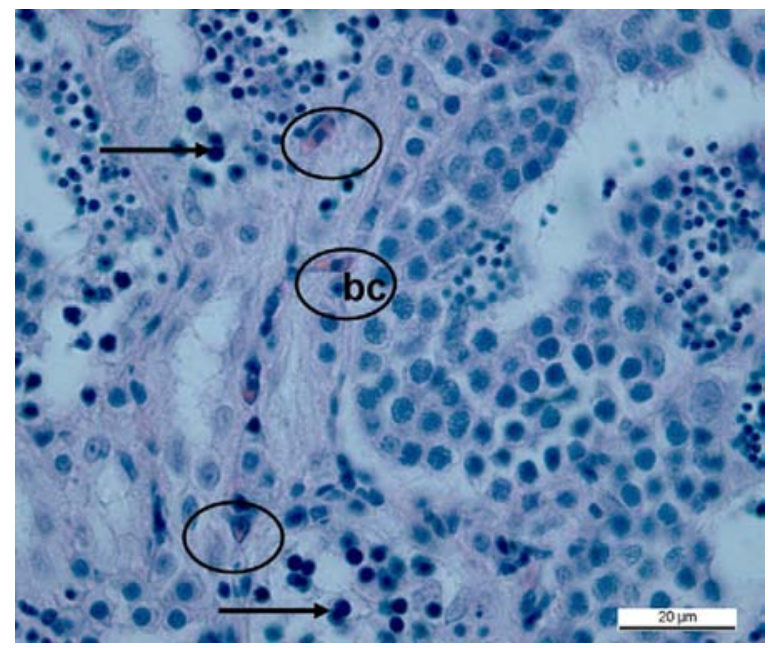

Fig. 13. The testes of an adult male $\mathbf{0}$. mossambicus from the 5 (xg/l exposure; stained with H\&E; Showing pyknosis (pk) in most of the spermatocytes. Characterised by condensation of the nuclei (arrow). Infiltration of red blood cells (bc) in the interstitial space highlighted by the circles. sis referred to the infiltration of red blood cells into the interstitial space.

\section{Discussion}

Endocrine disrupting chemicals are known to disturb the endogenous hormonal function of organisms, affecting behaviour and secondary sexual characteristics as well as the gonads. The purpose of this study was to examine changes in the gonads following exposure of 0. mossambicus to sub-lethal doses of DDT and how the exposure affected the offspring. There were no significant differences observed in the fecundity of organisms exposed to DDT and the control, suggesting that the concentrations applied in this study were not sufficient to cause a decrease in spawning ratios. The normal fecundity of tilapia has been investigated by various authors such as De Silva and Chandrasoma (1980) who reported that it varies from 360 to 1775 eggs per female for fish with length from 20 to $31.9 \mathrm{~cm}$ and weight ranging from 145 to $538 \mathrm{~g}$. According to Riedel (1965) the fecundity of the species ranges from 660 to 1754 eggs/100 g body weight. There were no indications that the applied DDT exposure in the present study had any adverse effects on the fecundity of the organisms.

There were decreased numbers of larvae reaching the end of yolk stage in the $5 \mathrm{jjig} / \mathrm{l}$ exposures. The developmental effects in the resulting larvae suggest assimilation of the chemical into the eggs of mature fish before spawning occurs (Donohoe and Curtis, 1996). The mortalities were attributed to the effects manifested during parental exposure, as these high mortalities were not occurring in the controls. Spawning was allowed to occur outside exposure conditions so the fertilized eggs had no direct contact with DDT water. Histological cross sections of 10dph larvae posterior to the buccopharyngeal cavity also suggested disturbance of structural features. During early differentiation there is a lot of mixing of cells during cleavage such that any one of the blastoderm cells can give rise to an unpredictable variety of tissue descendants. It is possible that such a stage is highly sensitive (Kimmel and Warga, 1987; Helde et al., 1994) to disruption by xenobiotics and abnormal tissue mixing can easily arise if there is interference. A study on zebrafish showed segmental scaffolding of chondroblasts and myoblasts suggesting that interactions between them coordinate their patterning in the embryo (Schilling and Kimmel, 1997). The observation in the controls indicated that malformations will also occur by chance or due to certain factors not accounted for in this investigation. In this study however DDT exposure was also a major contributing factor as shown by the significant levels in the exposed organisms. DDT exposure increased the incidence of larval skeletal deformities.

GSI is often applied as an endpoint of endocrine disruption because a reduced GSI usually points to a reduction in gonad mass. A reduction in testes mass leading to reduced GSI has been described as an estrogenic effect (Jobling et al., 1996; Christiansen et al., 1998; Pikulkaew and Wongsathein, 2006). Zaroogian et al. (2001) reported a dose-dependent atrophy of the testis in flounder treated with DDT. In females a reduced GSI of has been attributed to degeneration of ovaries (Scholz and Gutzeit, 2000). In this study there was no significant variation in the GSI of the breeding pairs between the fish exposed to DDT and the controls. The GSI did not serve as a sensitive endpoint for aqueous DDT exposure. A similar conclusion was drawn by Leaños-Castaneda et al. (2007) when there was no significant change in GSI in male tilapia exposed to o,p'-DDT.

In fathead minnow it has been shown that potential effects of steroidogenesis inhibitors include impaired vitellogenesis, pronounced oocyte atresia and suppression of oocyte maturation, ultimately leading to a reduction in fecundity (Ankley et al, 2002). While the occurrence of deformities was significant, fecundity was 
unaffected and ovarian lesions were not severe on a numerical scale. The main morphological characteristics of the ovary are the presence of a full range of oocyte maturation stages including oogo-nia, previtellogenic oocytes and mature vitellogenic oocytes (Van der Ven et al, 2003). The results from this study did not show a distinct interruption of the normal pattern of oocyte maturation which is normally indicative of significant inhibition of steroido-genesis. Inhibition of oocyte maturation is linked to inhibition of gonadotrophic hormone secretion (Wester et al., 2002).

Pre-ovulatory atretic follicles have been identified as a key histo-logical feature that shows EDC effects on ovarian development and spawning (Leino et al, 2005). Estrogenic exposure leads to relative increase in atretic follicles and a decrease of vitellogenic oocytes, intensity depending on the level of exposure (Van den Belt et al., 2002). The vitellogenic fluid which was observed, described as a proteinaceous (vitellogen rich) fluid (Van der Ven et al., 2003) is likely to be caused by degeneration of mature vitellogenic oocytes due to chemical exposure. Although there seemed to be a higher occurrence of alterations on the $5 \mathrm{u} . \mathrm{g} / \mathrm{l}$ group, out of a possible maximum score of 5 the alterations caused by DDT under our exposure conditions were not severe. It should also be pointed out that in all the samples though there were necrotic primary oocytes in some specimens, there was no evidence of suppression of the succession of oocyte maturation stages.

Spontaneous lesions of oocytes can also occur randomly under normal conditions, as reported in zebrafish studies, and this is a phenomenon of fish pathology that has been broadly investigated (Rossteuscher et al, 2008). Under the control conditions, the females did not show a susceptibility to spontaneous oocyte atresia, as shown by the low frequency of atresia, compared to exposed organisms. However it is important to note that under the conditions described in this study the females can eject their mature eggs when they are ready to spawn and incubate them in the mouth even in the absence of a male to fertilize them. It may be concluded that the prolonged absence of males may have been a source of stress which could have compromised the physiological reproductive health of the female fish. This stress may have caused the occurrence of atresia in control organisms. We could not conclude whether the necrotic oocytes were a direct result of DDT exposure since there was similar frequency in all the experimental groups.

It has been found in previous studies that DDT congeners act as estrogens impairing testes function via hormonal system (Pikulkaew and Wongsathein, 2006). It was evident that out of a possible maximum grade of 5 , the histological effects in the males were also generally not severe. The various stages of spermato-genesis could be identified in the cysts. The highest frequency of alterations occurred in the $5 \mathrm{u}$.g/l exposures. Some epithelial lifting and detachment of the basal membrane was observed in all the experimental groups and could not be directly linked to DDT exposure even though by comparison it occurred with higher frequency in the highest exposure concentration. Except for a single specimen showing signs of pyknosis, in which case the different types of cells contained in the cysts were not distinguishable, the males did not exhibit severe responses to DDT exposure. The changes in the histology of the testes are an indication of the disturbances to the HPG axis (Wester et al, 2002), but they did not affect ability to pair up with females and fertilize eggs. In the females however the effects were transferred to the next generation, therefore pointing to reduced reproductive success which impacts populations.

The present study demonstrated the effects of assimilation of DDT from the water column by fish and this having a mild effect on the parental gonads and direct effects on the F1 generation survival and viability. The use of $O$. mossambicus as a test species will provide specific information which can be compared with observations of effects on other species (Wester et al., 2002). The data from this paper is useful to assess the ecological risk of DDT in waters, based on the reproduction effects and the histopathological evidence.

\section{Conflict of interest statement}

None.

\section{Acknowledgements}

This project was supported by funding from the Water Research Commission of South Africa in collaboration with The University of Johannesburg and the University of Pretoria.

\section{References}

Ankely, G.T., Johnson, R.D., 2004. Small fish models for identifying and assessing the effects of endocrine-disrupting chemicals. ILAR 45 (4), $469-480$

Ankley, G.T., Kahl, M.D., Jensen, K.M., Hornung, M.W., Korte, J.J., Makynen, E.A., et al., 2002. Evaluation of the aromatase inhibitor fadrozole in a short-term reproduction assay with the fathead minnow (Pimephales promelas). Toxicol. Sci. 67, 121-130.

Arcand-Hoy, L.D., Benson, W.H., 1998. Fish reproduction: an ecologically relevant indicator of endocrine disruption. Environ. Toxicol. Chem. 17 (1), 49-57.

ATSDR, 2002. Toxicological Profile for DDT/DDD/DDE (update): US Department of Health and Human Services. Public Health Service. Agency for Toxic Substances and Disease Registry, Atlanta, GA.

Benton, M.J., Nimrod, A.C., Benson, W.H., 1994. Evaluation of growth and storage as biological markers of DDT exposure in sailfin mollies. Ecotoxicol. Environ. Saf. 29,1-12.

Bornman, M.S., Van Vuren,J.H., Bouwman, H., DeJager, T., Genthe, B., Barnhoorn, I.J., 2007. The use of sentinel species to determine the endocrine disruptive activity in an urban nature reserve. Water Research Commission. WRC Report No. 1505/1/07.

Bouwman, H., 2002. Malaria control and the paradox of DDT. Afr. - Environ. Wildlife 8, 54-56.

Cheek, A.O., Brouwer, T.H., Carroll, S., Manning, S., McLachlan,JA, Brouwer, M., 2001. Experimental evaluation of vitellogenin as a predictive biomarker for reproductive disruption. Environ. Health Perspect. 109,681-690.

Christiansen, T., Korsgaard, B., Jespersen, A., 1998. Effects of nonylphenol and 17 beta-oestradiol on vitellogenin synthesis, testicular structure and cytology in male eelpout (Zoarces viviparous). J. Exp. Biol. 201,179-192.

Dalvie, M.A., Myers, J.E., Thompson, M.L., Dyer, S., Robins, T.G., Omar, S. Riebow, J., Molekwa, J., Kruger, P., Millar, R., 2004. The hormonal effects of long-term DDT exposure on malaria vector-control workers in Limpopo Province, South Africa. Environ. Res. 96, 9-19.

De Silva, S.S., Chandrasoma, J., 1980. Reproductive biology of Sarotherodon mossambicus, an introduced species, in an ancient man-made lake in Sri Lanka. Environ. Biol. Fishes 5, 250-253.

Donohoe, R.E., Curtis, L.R., 1996. Estrogenic activity of chlordecone, o,p'-DDT and o,p'-DDE in juvenile rainbow trout: induction of vitellogenesis and interaction with hepatic estrogen binding sites. Aquat. Toxicol. 36,31-52.

Du Preez, H.H., van der Merwe, M., van Vuren, J.H.J., 1997. Bio-accumulation of selected metals in African sharptooth catfish Clarias gariepinus from the lower Olifants River, Mpumalanga, South Africa. Koede 40 (1), 77-90.

Edmunds, J.S.G., Mccarthy, R.A., Ramsdell, J.S., 2000. Permanent and functional male-to-female sex reversal in d-rR strain medaka (Oryzias latipes) following egg microinjection of o,p'-DDT. Environ. Health Perspect. 108 (3), 219-224.

Gisbert, E., Rodriguez, A., Castello-Orvay, F., Williot, P., 1998. A histological study of the development of the digestive tract of Siberian sturgeon (Acipenser haeri) during early ontogeny. Aquaculture 167,195-209.

Guillete, L.J., Gross, T.S., Masson, G.R., Matter, J.M., Percival, H.F. Woodward, A.R., 1994. Developmental abnormalities of the gonad and abnormal sex hormone concentrations in juvenile alligators from contaminated and control lakes in Florida. Environ. Health Perspect. 102,680-688.

Hall, L.C., Okihiro, M., Johnson, M.L., Teh, S.J., 2007. Surflan ${ }^{\mathrm{TM}}$ and oryzalin impair reproduction in the teleost medaka (Oryzias latipes). Mar. Environ. Res. 63, 115-131.

Hatikakoty, G., Biswas, S.P., 2004. Studies on certain aspects ofthe reproductive biology of Mouth-brooding tilapia, Oreochromis mossambicus (Peters) from Assam, India. Available: http://ag.arizona.edu/azaqua/ista/ista6/ista6web/pdf/112.pdf.

Helde, K.A., Wilson, E.T., Cretekos, C.J., Grunwald, D.J., 1994. Contribution of early cells to the fate map ofthe zebrafish gastrula. Science 265,17-20. 
Hill, J., 2009. Effects of heptachlor and DDE on the GH-IGF axis in female tilapia, Oreochromis mossambicus. JYI19 (9).

Holden, K.K., Bruton, M.N., 1992. A life history approach to the early ontogeny ofthe Mozambique tilapia Oreochromis mossambicus (Pisces, Cichlidae). S. Afr. J. Zool. 24, 173-191.

Hutchinson, T.H., Ankley, G.T., Segner, H., Tyler, C.R., 2006. Screening and testing for endocrine disruption in fish-biomarkers as "signposts," not "traffic lights," in risk assessment. Environ. Health Perspect. 114,106-114. 
Jobling, S., Sheahan, D., Osborne, JA, Matthiessen, P., Sumpter, J.P., 1996. Inhibition of testicular growth in rainbow trout (Oncorhynchus mykiss) exposed to estrogenic alkylphenolic chemicals. Environ. Toxicol. Chem. $15,194-202$

Kelce, W.R., Stone, C.R., Laws, S.C., Gray, L.E., Kemppalnen, J.A., Wilson, E.M., 1995. Persistent DDT metabolite p,p'-DDE is a potent androgen receptor antagonist. Nature 357, 581-585.

Kimmel, C.B., Warga, R.M., 1987. Indeterminate cell lineage of the zebrafish embryo. Dev. Biol. 124, 269-280.

Leaños-Castañeda, O., Van Der Kraak, G., Rodríguez-Canul, R., Gold, G., 2007. Endocrine disruption mechanism of o,p'-DDT in mature male tilapia (Ore-ochromis niloticus). Toxicol. Appl. Pharmacol. 221,158-167.

Leino, R.L., Jensen, K.M., Ankley, G.T., 2005. Gonadal histology and characteristic histopathology associated with endocrine disruption in the adult fathead minnow (Pimephales promelas). Environ. Toxicol. Pharmacol. 19, 85-98.

Marchand, M.J., Pieterse, G.M., Barnhoorn, I.E.J., 2008. Preliminary results on sperm motility and testicular histology of two feral fish species, Oreochromis mossam-bicus and Clarius gariepinus from a currently DDT-sprayed area, South Africa. J. Appl. Ichthyol. 24, 423-429.

Metcalfe,T.L., Metclafe, CD., Kiparissis, Y., Niimi, A.J., Foran, CM., Benson, W.H., 2000. Gonadal development and endocrine responses in Japanese medaka (Oryzias latipes) exposed to o,p'-DDT in water or through maternal transfer. Environ. Toxicol. Chem. 19,1893-1900.

Papoulias, D.M., Villalobos, S.A., Meadows, J., Notlie, D.B., Giesy,J.P., Tillitt, D.E., 2003. In ovo exposure to o,p'-DDE affects sexual development but not sexual differentiation in Japanese medaka (Oryzias latipes). Environ. Health. Perspect. 111, 29-32.

Patyna, P.J., Davi, R.A., Parkerton, T.F., Brown, R.P., Cooper, K.R., 1999. A proposed multigeneration protocol for Japanese medaka (Oryzias latipes) to evaluate effects of endocrine disruptors. Sci. Total Environ. 233, 211-220.
Pikulkaew, S., Wongsathein, D., 2006. The effect of DDT congeners on reproductive function in male guppy (Poecilia reticulata). CUMJ 5 (2), 219-225.

Riedel, D., 1965. Some remarks on the fecundity of Tilapia (J. mossambica Peters) and its introduction into Middle Central America (Nicaragua) together with a 
first contribution towards the limnology of Nicaragua. Hydrobiologia 25, 357-

388. Rogan, W.J., Chen, A., 2005. Health risks and benefits of

bis(4-chlorophenyl)-1,1,1-

trichloroethane (DDT). Lancet 366, 763-773. Rossteuscher, S

Schmidt-Posthaus, H., Schäiers, C, Teigeler, M., Segner, H., 2008.

Background pathology ofthe ovary in a laboratory population of zebrafish (Danio

rerio). Dis. Aquat. Org. 79,169-172. Schilling, T.F., Kimmel, C.B., 1997.

Mucoskeletal patterning in the pharyngeal segments ofthe zebrafish embryo.

Development 124, 2945-2960. Scholz, S., Gutzeit, H.O., 2000.

17-Alpha-ethinylestradiol affects reproduction, sexual reproduction, sexual differentiation and aromatase gene expression ofthe medaka (Oryzias latipes). Aquat. Toxicol. 50,363-373. Turusov, V.,

Rakitsky, V., Tomatis, L., 2002. Dichlorodiphenyltrichloroethane (DDT): ubiquity, persistence, and risks. Environ. Health Perspect. 110 (2),

125-128. US Environmental Protection Agency, 2006. Fish Screening Assay

Discussion Paper 4/13/2006. US Environmental Protection Agency, Washington, DC, www.epa.

gov/endo/pubs/edmvac/fish_assay_discussion_paper_041306.pdf. Van den

Belt, K., Wester, P., van der Ven, L.T.M., Verheyen, R., Witters, H., 2002. Time-dependent effects of ethynylestradiol on the reproductive physiology in

zebrafish (Danio rerio). Environ. Toxicol. Chem. 21, 767-775. Van der

Ven, L.T.M., Wester, P.W., Vos, J.G., 2003. Histopathology as a tool for the evaluation of endocrine disruption in zebrafish (Danio rerio). Environ. Toxicol.

Chem. 22, 908-913. Van Dyk,J.C, Pieterse, G.M., van Vuren,J.H.J., 2007.

Histological changes in the liver

of Oreochromis mossambicus (Cichlidae) after exposure to cadmium and zinc.

Ecotoxicol. Environ. Saf. 66, 432-440. Wester, P.W., van derVen,

L.T.M.,Vethaak,A.D., Griwis, G.C.M., Vos, J.G., 2002. Aquatic

toxicology: opportunities for enhancement through histopathology.
Environ.

Toxicol. Pharmacol. 11, 289-295. Zaroogian, G., Gardner, G., Horowitz, D.B., Gutjahr-Gobell, R., Haebler, R., Mills, L., 2001. Effect of 17beta-estradiol, o,p'-DDT, octylphenol and p,p'-DDE on gonadal

development and liver and kidney pathology in juvenile male summer flounder

(Paratichthys dentatus). Aquat. Toxicol. 54,101-112. 